\title{
Marché européen des margarines et des matières grasses à tartiner
}

Inneke HERREMAN

IMACE, International Margarine Association of the Countries of Europe, Avenue de Tervuren, 168 box 12, B-1150 Brussels,

Tél. : + 32277233 53,

Fax : + 3227714753

<imace.ifma@imace.org>

\begin{abstract}
IMACE, the International Margarine Association of the Countries of Europe represents 21 national associations of the margarine industry. While in 2003 the World Production increased to 10.1 Mt (9.3 Mt in 1998), the production of the European countries of 15 was 1.9 Mt (2.3 Mt in 1998). In 2003 the most important European producing countries are Germany (446,000 t), the United Kingdom $(322,000 t)$, Belgium $(268,000 t)$, the Netherlands $(289,000 t)$ and Poland $(510,000 t)$. The changes in food habits involved a decline of consumption. But the sector has invested a lot in optimizing the composition of its products, with a special attention for unsaturated fatty acids. European Union food legislation that has a particular impact on the margarine industry are the proposed EU Claims regulation, the proposed EU harmonization of vitamins and minerals, the EU directives on full ingredient labelling and on nutrition labelling. But the industry also actively pursues a road of self-regulation i.e. IMACE Code of Practice on Nutrition Claims, IMACE Code of Practice on Addition of Vitamins, IMACE Code of Practice on TFA. Several scientific publications recognize the margarine industry's efforts in reducing TFA and underline that they are known as a determinant factor in the overall reduction of TFA in food products.
\end{abstract}

Key words: IMACE, margarine, European market, food habits

en Pologne de $390000 \mathrm{t}$ (1998) à $510000 \mathrm{t}$ (2003).

En 2003, les principaux pays européens producteurs sont l'Allemagne avec $446000 \mathrm{t}$, le Royaume-Uni avec $322000 \mathrm{t}$, la Belgique avec 268000 t, les Pays-Bas avec 289000 t et la Pologne avec $510000 \mathrm{t}$. La France a vu sa production diminuer de 138000 t en 1998 à 75000 t en 2003, alors que les ventes s'élèvent à 157000 t en 2003.

Les autres grands producteurs en 2003 sont le Brésil (493000 t), le Japon (177 000 t), la Turquie (475 $000 \mathrm{t}$ ) et les États-Unis (960000 t).

\section{Structure de l'industrie de la margarine}

Depuis quelques années, le secteur est marqué par une centralisation croissante de la production et par une consolidation de l'industrie. Les changements dans les habitudes alimentaires ont amené un déclin de la consommation. Mais le secteur a beaucoup investi dans une optimalisation de la composition de ses produits, avec une attention toute particulière portée sur les graisses insaturées. Par ailleurs, ces dernières années ont été marquées par l'essor de nouveaux produits enrichis en stérols/stanols végétaux, réduisant activement le taux de cholestérol.

\section{Réglementation européenne}

En matière de législation européenne, les principaux textes ayant un impact direct sur le secteur de la margarine sont le Règlement 2991/94 sur les matières grasses à tartiner, la
Proposition de règlement sur les allégations, la Proposition de règlement sur l'addition des nutriments, la Directive sur l'étiquetage nutritionnel et les Dispositions sur l'étiquetage des allergènes.

\section{Règlement sur les matières grasses tartinables}

Le Règlement 2991/94 établit les « dénominations de vente » pour le beurre, la margarine et la matière grasse composée selon leur contenu de matière grasse (MG) :

- $80 \%-90 \%$ MG (margarine) ;

- $60 \%-62 \%$ MG (trois-quarts margarine) ;

- 39 \%-41 \% MG (demi-margarine) ;

- Matière grasse à tartiner $x \% \mathrm{MG}$.

Après de longues années de discussion avec les États membres et avec les secteurs margarinier et laitier, le Règlement 2991/94 fut l'aboutissement d'une demande de l'industrie ellemême!

Il détermine également la terminologie spécifique de la «teneur réduite en matière grasse » ou « allégé » pour les produits de 41 \%-62 \% MG et «à faible teneur en matière grasse/light/léger » pour les produits inférieurs à $41 \%$. Autres obligations d'étiquetage spécifique : I'indication du pourcentage de MG et le pourcentage en sel.

En juillet 2002, un rapport de la Commission européenne a confirmé la terminologie du Règlement 2991/94 c'est-à-dire l'utilisation de « à faible teneur en matière grasse/light/léger » pour les produits inférieurs à $41 \% \mathrm{MG}$. Toutefois, à terme, cette terminologie devra être adaptée au Règlement sur les allégations nutritionnelles, qui propose un maximum de $3 \mathrm{~g}$ 
MG/100 g pour l'utilisation du terme « à faible teneur en matière grasse »!

\section{Allégations}

En juillet 2003, la Commission européenne a fait une proposition pour l'harmonisation des allégations nutritionnelles et de santé. Les principaux éléments de la proposition sont :

- Une restriction générale sur base des « profils nutritionnels » (tenant compte de la quantité de MG, AGS, AGT, sucre, sel).

- Une condition générale: une justification scientifique.

- «Allégations nutritionnelles » sur base de la liste Codex

- " Allégations de santé » sur base d'une liste à établir. À cet effet, les États membres devront communiquer la liste des allégations de santé approuvées au niveau national, décrivant le rôle généralement reconnu d'un nutriment c'est-à-dire : «le calcium fortifie les os ». Et dans les 3 ans la Commission EU proposera une liste consolidée.

- «Allégations concernant la réduction d'un risque de maladie » exigent une autorisation préalable, aussi bien du dossier scientifique que du libellé : « le calcium réduit l'ostéoporose »; - Allégations nutritionnelles concernant MG : - « à faible teneur en $M G$ » $(\max 3 \mathrm{~g} / 100 \mathrm{~g})$ - « à teneur réduite (en $M G)$ »/« light »/allégé (min $30 \%$ de réduction)

- "à faible teneur en AGS " (max $1,5 \mathrm{~g} / 100 \mathrm{~g})$.

Depuis le début du débat européen, les principaux éléments de l'action IMACE ont été de :

- Demander une exemption pour le Règlement 2991/94.

- Demander l'inclusion des allégations nutritionnelles « riche en PUFA/MUFA/UFA/Oméga 3 , source Oméga 3 et sans cholestérol » sur base des critères du Code de pratique IMACE. - Mener des actions auprès des membres du Parlement européen et des représentants nationaux au groupe de travail du Conseil.

Les demandes IMACE ont été introduites dans la position CIAA et dans le Projet du Rapport PE, ainsi que dans le débat au Conseil CE.

La discussion au Conseil européen s'est focalisée sur le champ d'application, les profils nutritionnels, la procédure d'autorisation, la liste des allégations nutritionnelles. La Commission européenne a demandé l'avis EFSA concernant les conditions pour "riche en PUFA/ MUFA/UFA/Oméga 3 ». Le rapport EFSA est attendu mi-2005.

Au Parlement, le Comité Environnement adoptera le Rapport de Mme Poli-Bortone en avril 2005 et l'adoption en session plénière est prévue en mai 2005. La deuxième lecture de la proposition se fera en 2006.
Depuis 1992, IMACE a adopté un Code de pratique sur les allégations nutritionnelles. Considérant les développements du marché, les connaissances actuelles concernant graisses/ santé et la proposition EU, le code IMACE a été revu en 2003. Il établit des conditions reconnues scientifiquement, justifiées du point de vue nutritionnel et calculés sur base d'une consommation moyenne de margarine/ matière grasse tartinable de $20 \mathrm{~g}$ par jour.

\section{Addition des nutriments}

En novembre 2003, la Commission européenne a proposé I'harmonisation de l'ajout volontaire des vitamines/minéraux (sur base $d^{\prime} u n e$ liste établie) et autres substances (liste ouverte), avec étiquetage nutritionnel obligatoire. Toutefois, dans la proposition, les mesures nationales d'adjonction obligatoire ne sont pas interdites. Un règlement ultérieur déterminera les limites maximales sur base d'évaluations EFSA.

Actuellement, l'addition des vitamines dans les margarines est conditionnée par une législation nationale très divergente : Belgique (obligatoire), France et Danemark (restriction vitamine D), Suède (obligatoire), Royaume-Uni (obligatoire seulement pour margarine, mais pas pour une matière grasse tartinable), PaysBas (avant obligatoire, mais maintenant accord volontaire), etc.

En mai 2004, IMACE adoptait un Code de pratique concernant les vitamines A-D. Ce code confirme les raisons nutritionnelles pour l'ajout de vitamines A-D dans les margarines. En effet, la présence naturelle de vitamines A-D dans les denrées alimentaires est limitée. Par ailleurs, la protection solaire et la réduction dans la consommation de MG influencent négativement I'apport de vitamines A-D. Le code concerne principalement les produits au détail, mais fait référence aux produits industriels s'ils sont fortifiés.

Le code IMACE établit des limites maximales basées sur les avis EFSA et qui sont nutritionnellement pertinentes pour une consommation moyenne de $20 \mathrm{~g}$ par jour de margarine c'està-dire $800 \mu \mathrm{g}$ de vitamine $\mathrm{A}$ par $100 \mathrm{~g}$ de produit fini et $7,5 \mu \mathrm{g}$ ou $10 \mu \mathrm{g}$ (Scandinavie) de vitamine $\mathrm{D}$ par $100 \mathrm{~g}$ de produit fini.

\section{Étiquetage nutritionnel et acides gras trans}

En mars 2003, le Danemark a introduit une loi établissant une limite de $2 \%$ d'acides gras trans (AGT) dans les produits alimentaires. Alors que pour les margarines de table la limite de $2 \%$ maximum s'applique à partir du 1/06/2003, pour les margarines industrielles la limite de $2 \%$ s'applique à partir du 1/01/2004. La limite de $2 \%$ ne concerne ni les AGT laitiers, ni les exportations...

Suite aux mesures unilatérales danoises, la Commission européenne a demandé l'avis de I'EFSA. Dans son rapport publié en septembre 2004, EFSA a conclu qu'il n'y avait pas lieu de faire une distinction entre les AGT naturels et industriels, ni d'imposer des limites puisque le problème des acides gras saturés est bien plus important. Que la définition AGT couvre aussi bien les AGT industriels que naturels est également confirmée aussi bien par l'Afssa que par le Codex Alimentarius.

II est dès lors fort possible que la future révision de la Directive EU concernant l'étiquetage nutritionnel introduise de façon obligatoire l'étiquetage des AGS et AGT. La révision est prévue pour 2006.

Déjà en 1995, IMACE avait adopté un Code de pratique afin de réduire à $5 \%$, niveau moyen AGT dans le beurre, les AGT dans les produits margarines. Une révision du Code de pratique en juin 2002 a introduit pour les margarines de table un maximum de $1 \%$ AGT, basé sur le produit et maximum $5 \%$ AGT pour les matières grasses composées. Pour les margarines industrielles, l'objectif est de $5 \%$ AGT, tout en tenant compte des exigences de fonctionnalité et des spécifications commerciales.

L'industrie s'engage à encourager ses clients du bien fondé des margarines industriels à faible teneur de AGT et de présenter des margarines alternatives à max $5 \%$ AGT. Par ailleurs, il est convenu de ne pas augmenter le total AGS/AGT et de continuer les efforts de réduction à long terme.

Plusieurs publications scientifiques soulignent les efforts de l'industrie de la margarine, qui sont reconnus comme un facteur déterminant dans la réduction des AGT.

\section{Étiquetage des allergènes}

La Directive EU 2003/89 impose la déclaration complète des ingrédients à partir de 25/11/04, visant surtout l'étiquetage des allergènes. Toutefois, une procédure d'exemption est prévue pour les produits dérivés de substances allergéniques.

Sur base des dossiers scientifiques soumis à I'EFSA, la Commission européenne a adopté une liste d'exemptions provisoires (valables jusqu'au 25 novembre 2007), incluant notamment l'huile de soja raffinée pour lequel FEDIOL et IMACE ont soumis un dossier conjoint.

La demande d'exemption permanente devra, elle, se faire sur base d'un nouveau dossier nécessitant encore davantage de recherches analytiques et cliniques, qui seront financées par les industries de l'huile et de la margarine. Le nouveau dossier sera présenté dans le courant de 2006. 